\title{
Serial Concatenation of Quadratic Interleaved Codes in Different Wireless Doppler Environments
}

\author{
Ardavan Rahimian, Farhad Mehran \\ School of EECE \\ University of Birmingham \\ Birmingham B15 2TT, West Midlands, UK \\ \{rahimian \& farhad.mehran\}@ieee.org
}

\author{
Robert G. Maunder \\ School of ECS \\ University of Southampton \\ Southampton SO17 1BJ, Hampshire, UK \\ rm@ecs.soton.ac.uk
}

\begin{abstract}
The remarkable development of mobile networks has enabled ubiquitous communications that has transformed the way people connect with each other. Meanwhile, the growing adoption of smartphones, tablets, and increasingly bandwidthintensive applications and services is driving unprecedented mobile broadband traffic growth; therefore, finding state-of-theart strategies for improving the wireless transmission reliability has emerged significantly. In particular, to enable significant mitigation of the detrimental effects of multipath fading, highperformance forward error correction (FEC) techniques have now recognized to become a vital part of modern digital wireless systems. This work is dedicated to address a class of highperformance iteratively decoded FECs referred to as serial concatenation of quadratic interleaved codes (SCQICs) wherein the reordering arrays for permutor and unscramble in the FEC encoding/decoding entities enjoy the remarkable algorithm proposed by Takeshita et al. seminal contribution which: (1) yields provisioning coding gain in both waterfall and error-floor regions of the bit error rate (BER) performance curve (2) enables possibility of analysis and compact representation, (3) requires straightforward implementation. We have observed that their utilization for the modern wireless communications applications remained scarce due to the lack of strong and comprehensive performance predictions over the different statistical wireless channel models. Hence, in this paper, we endeavor to analyze and examine the error-correction capability of SCQICs vastly from the various aspects, and investigate their resultant coding gains in different wireless Doppler environments.
\end{abstract}

Index Terms-concatenated codes, Doppler spectrum, forward error correction (FEC), interleaver, permutation, turbo code, wireless communications.

\section{BACKGROUND}

\section{A. Introduction}

In the past two decades, area of the FECs has been revolutionized by the practical realization of the capacityapproaching coding schemes initiated by the breakthrough invention of turbo code family and their associated decoding algorithms introduced in [1-2]. A few years after the invention of classic turbo coding schemes based on a rate $1 / 2$ parallel $16-$ state convolutional codes which have shown to achieve error correction characteristics close to the Shannon-limit at a BER of $10^{-5}$ (at $E_{b} / N_{0}=0.7$ ) provided that the MAP decoder with 18 iterations and large interleaver of 65536 bits are used, researchers became aware that their common irreducible errors which appear due to their low distance at BER $\leq 10^{-5}$ (note that this specific point varies between different designs) could be eliminated and improved (i.e. resulting steeper slope of the error-rate curve when computed as a function of $E_{b} / N_{0}$ ) by employing a variation of classic turbo codes, conventionally referred to as serial concatenation of interleaved codes (SCICs) [3-4].

In addition to the concatenation scheme, the structure of the permutor, in terms of type and depth, play a key role in determining performance of a given turbo code. In particular, while the design of interleavers for the parallel turbo codes has enjoyed significant attention, interleavers designed for SCICs remain scarce [5]. This may be because, in contrast to the parallel turbo codes, SCICs typically employ a concatenation of different codes, having different distance properties that complicate the interleaver design [5]. Based on the available literature, it has been reported that all turbo codes (independent of concatenation category), can achieve an outstanding highperformance gain by utilizing the sufficiently large interleaver /deinterleaver lengths (e.g. Berrou's 65536-bit parallel scheme [1]); however, the long buffering/processing delays caused by such a large depth is often too prohibitive for providing high data throughput in various applications of modern wireless systems. In order to accommodate such demands, there have been some successful attempts in designing the turbo code permutations of feasible size, suitable for short-frame wireless transmission applications; however, the resultant coding gains are not large compared with large depth cases, and therefore, further investigations and developments are still open in order to find well-designed permutations.

\section{B. Related Works}

In [6-7], Takeshita et al. proposed a new deterministic permutation structure with a very simple representation based on the quadratic congruences over the ring of integers modulo powers of two, that have structure which allows the possibility of analysis (unlike the randomly generated permutations which suffer from significant lack of adequate analysis and compact representation), as well as the straightforward implementation. Since for the effective design of FECs, the problem of channel modeling is crucial, that has motivated us to employ the interesting characteristics of Takeshita et al. permutations in the SCIC-coded wireless communication systems in different Doppler environments; i.e. taking into account the frequency 
dispersion of the channel by utilizing the different well-known Doppler spectra for reflecting statistical channel characteristics.

In [8], we have developed a class of SCICs, wherein the permutor/unscrambler entities enjoy utilization of the Takeshita et al. algorithm for improving the error-rate performance of wireless systems with BPSK over Alamouti's space-time coded [9] MISO fading channels, when line-of-sight (LoS) exists in the propagation path and under the assumption that the spectral broadening of received signal follows Bell spectrum proposed in IEEE 802.11 TGn model. In order to provide a good solution in terms of both bandwidth and power efficiency, in [10], we extended the previously introduced technique for the multilevel QAM transmissions with arbitrary number of transmitter and receivers based on Tarokh et al.'s orthogonal space-time block codes (OSTBCs) [11-12], under the same Doppler spectra but with the blocked LoS path in the direction of propagation for undertaking the worst case radio channel scenario, wherein the statistical amplitude of the received signal's envelope follows Rayleigh probability density function (PDF). As far as the randomization in the time order of the bit sequences at the permutor/unscrambler components of the FEC encoder/decoder sides is performed based on the quadratic congruence [6-7], we referred to this class of the SCICs as serial concatenation of quadratic interleaved codes (SCQICs).

In [13], performance of the SCICs over generic incoherent SISO aeronautical channels has been analyzed and presented in a semi-tutorial manner. The main challenges in the design of the SCICs have been addressed, and optimal and suboptimal performance parameter combinations have been utilized in order to analyze the error-rate performance of the generic system and compute the approximated coding gain; however, we found out that the performance predictions need further concentration. In particular, recently introduced and matured techniques for compensating the worst case channel conditions should be integrated into the classic SCIC encoder/decoder entities; hence in [14], we have analyzed the BER performance of the SCQICs (i.e. employed Takeshita et al. interleavers in classic SCICs) and integrated the resultant FEC with the spacetime codes of the Alamouti's twin-antenna. The investigations have been run over the MISO stochastic aeronautical channels using the coherent and incoherent phase-shift-keying (PSK) modulations, and the energy gaps for the systems with coherent and incoherent PSK schemes have been then presented for the $\mathrm{BER} \approx 10^{-1.5}-10^{-5}$.

\section{Scientific Contribution of This Article}

In this paper, we aimed to endeavor the following issues for the effective design of the SCQICs:

(1) Propose the detailed construction and the algorithmic procedure for design of FEC-encoder/-decoder. We particularly tackled the short-block SCQICs, since there have been much interests for employing short message-blocks in spite of their significant inferior coding gains; that is due to the fact that for accommodating the explosive demands for today's anytime anywhere communications, employing FECs with high delays are prohibited.

In addition, the main outcomes with the design issues and performance investigations of our previous works [8,10,14], have also been addressed briefly through the short examples.
(2) In order to provide a concrete demonstration of the outstanding coding gains of the SCQICs for wireless channels, in this work, the required SNRs for provisioning the BER $\approx 10^{-5}$ have been computed comprehensively for the different wireless Doppler environments, over the Rayleigh-faded SISO, MISO, and MIMO links (which will be detailed in section V).

(3) As for the case of classic Berrou et al. FECs, the exceptional coding gains and overall decoding complexities of SCIC-coded systems heavily depend on the iterative decoding algorithm employed for the soft-decision component decoders (and therefore the same level of importance for SCQICs). Since high buffering/processing delays of SCQIC's iterative decoder is a major disadvantage for these types of FECs, the SCQICs are developed for the original and also approximated iterative MAP turbo decoders (i.e. BCJR-MAP [15], BCJR-Log-MAP [16] and BCJR-Max-Log-MAP [17]) to make the design issues associated with the error-correction capabilities and resultant complexities (which in turn increases the decoding processing /buffering delays) trade-off more convenient; i.e. to enable higher level of the code selection flexibility in error-rate versus computational complexity trade-off which is of great crucial importance in the PHY layer of almost all the modern wireless communication systems.

Hence, in this contribution, we are going to tackle most of the key aspects (circled with the dashed lines) of the design of attractive FEC entity summarized by Hanzo et al. in Fig. 1 [1819]. We will distinguish our scientific contributions relative to the prior works wherever we come up with the more detailed specifications rather than this section's brief outlines. In order to avoid unrealistic simulation conditions, the fixed factors have been selected so that they result in moderate contribution in operating points of the overall PHY layer performance tradeoffs, well-known universally as the promising generic fixedparameters at the FEC design stage e.g. spectrally efficient high throughput 8-DPSK modulation has been utilized throughout the numerical performance evaluations.

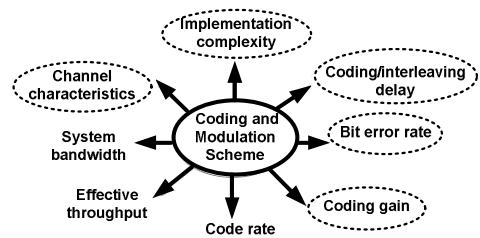

Figure 1. Factors affecting the design of FECs.

\section{SYSTEM MODEL}

\section{A. System Description}

In Fig. 2, the SCQIC-coded discrete-time system model is depicted. A wireless system is considered where transmitters and receivers are either of single or multiple omnidirectional antennas. Message bits are first FEC-encoded by the SCQIC encoder (which will be detailed in section III), and afterwards, inserted into differential encoder. In spite of higher required $E_{b} / N_{0}$ when differential coding (DC) is employed for attaining the same error-rate, we utilize DC before forming the signal constellations for pre-compensating the common phase errors of wireless communications (discussed in [20-21]). Afterwards, 
differentially encoded bit sequences are modulated by $M=8$ DPSK modulator. Herein, the constellation formation enjoys mapping as depicted in Fig. 2, which results in considerable coding gain over binary-coded constellations. The transmission sequences for the space-time encoding for the twin-antenna and triplet-antenna systems are given in (32) and (37) of [12].

At the reception side the decision criteria expressed in (17)(20) of [9] are used for detecting symbols of the system with two transmitting antennas; meanwhile the maximum-likelihood (ML) detector amounts to minimize the decision metrics given in appendix of [11] for detecting symbols of the system with three transmitting antennas. From the received wireless signal constellations of 8-PSK signaling over the single-input singleoutput (SISO), $2 \times 1$ multiple-input single-output (MISO), $2 \times 2$ and $3 \times 3$ multiple-input multiple-output (MIMO) links of next section - part B; the detected symbols have been inserted into the DC decoder for performing the reverse function applied at transmitter side. For recovering the information bit sequences, the output of DC decoder is fed into the iterative SCQIC decoder (which will be detailed in section IV).

\section{B. Channel Model}

The problem of modeling channels is crucial for effective design of FECs. Considering the notion of universality with respect to the channel parameters for designed FECs, it is indispensable to generalize and observe the behavior of their extended variants for commonly used wireless links; hence, the reference channel models are employed which establish reproducible channel conditions for assessing the capability of the designed technique for maximizing the capacity and also improving performance in a manner that is unified and wellcooperated on by many parties; meanwhile, to some extent, answer the key question, "Is the coding scheme capable of eradicating bit errors over the near practical channels that the transmissions are going to be carried out in reality?". This key question is behind vast investigations when one proposes the power efficient FEC schemes and the related research community dedicate intensive efforts in order to characterize the performance of the proposed scheme over various different wireless channels.

In order to assess the system performance accurately, while for planning and deployment the location-specific models are used, the stochastic models are more commonly used for the design, testing, and type approval [22]. For describing the statistical time-varying nature of the received wireless signal's amplitude when mobile wireless system with blocked LoS is moving the characteristics of urban propagation environments, Rayleigh distribution is the most commonly used model (at the system design phase) for predicting the PDF of entries of the channel matrix (CM). Denote the noisy superposition of the transmitted signals appeared at the receiver by $y=H . c+\gamma$ where $y=\left[y_{1}, \ldots, y_{N_{r}}\right]^{T}$ is an $N_{r}$-element vector of the received signals, $H$ is an $\left(N_{r} \times N_{t}\right)$-element $\mathrm{CM}$ i.e. $c=\left[c_{1}, \ldots, c_{N_{t}}\right]^{T}$ which is an $N_{t}$-element vector of the transmitted signals, and $\gamma=\left[\gamma_{1}, \ldots, \gamma_{N_{r}}\right]^{T}$ is an $N_{r}$-element noise vector of the entries of the additive white Gaussian noise (AWGN) process having a zero mean and a variance of an $N_{0} / 2$ per dimension.

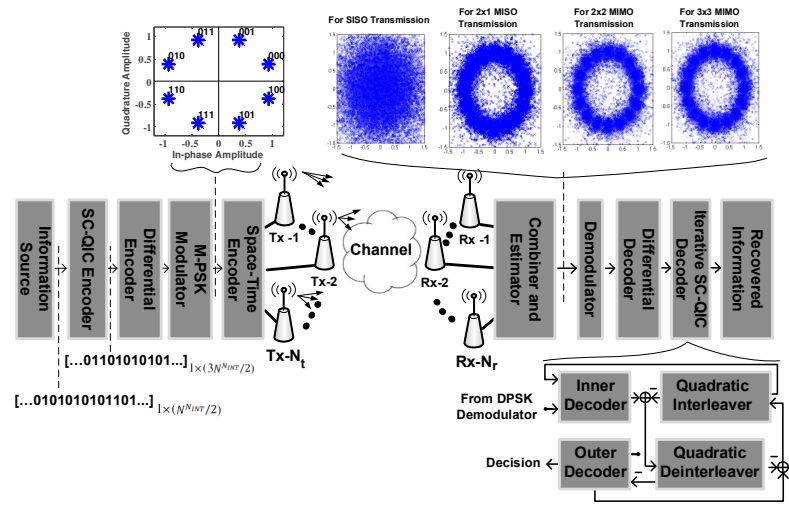

Figure 2. Schematic of the SCQIC-coded wireless system.

In addition, the Doppler power spectral density (PSD) is the key parameter in the description of channel [23] since that has crucial contribution in the statistical descriptions of the received spectrum broadenings. The Rounded, Gaussian, BiGaussian and Bell Doppler PSD functions are widely accepted for various applications and channel conditions summarized in Table I. For providing broad investigations for the SCQIC error-rates in the provisioning region i.e. $\approx 10^{-5}$ (presented in section V), simulations consider the different multiple-antenna scenarios based on the assumption of i.i.d. Hence, for every multiple-antenna system configuration (from $N_{t}, N_{r} \in\{2,3\}$ ) with multipath Rayleigh-faded wireless propagation channel, distinct performance evaluation process has been conducted for each Doppler PSD model.

TABLE I. DOPPlER SPECTRA FOR Single-/MultiPle-ANTENNA RAYLEIGH PROCESSES OF THE CHANNEL SCENARIOS.

\begin{tabular}{|c|c|c|c|}
\hline $\begin{array}{c}\text { Spectral } \\
\text { Broadening } \\
\text { Model }\end{array}$ & System Model & Description & Applications \\
\hline Rounded & $\begin{array}{l}\text { Channel models } \\
\text { for fixed wireless } \\
\text { applications [24]. }\end{array}$ & $\begin{array}{c}S(f) \\
=\left\{\begin{array}{c}1-1.72 f_{0}^{2}+0.78 f_{0}^{4}\left|f_{0}\right| \leq 1 \\
0 \quad\left|f_{0}\right|>1\end{array}\right. \\
\text { * where } f_{0}=f / f_{m} \text { and } f_{m} \\
\text { is a maximum Doppler } \\
\text { frequency. }\end{array}$ & $\begin{array}{l}\text { Proposed for the scatter } \\
\text { component of fixed } \\
\text { wireless channels. }\end{array}$ \\
\hline Gaussian & $\begin{array}{c}\text { ANSI J-STD-008 } \\
\text { reference channel } \\
{[25] .}\end{array}$ & $S(f)=\frac{1}{\sqrt{2 \pi \sigma^{2}}} \exp \left(-\frac{f^{2}}{2 \sigma^{2}}\right)$ & $\begin{array}{l}\text { Proposed for the PCS } \\
\text { applications; also, for the } \\
\text { aeronautical channels } \\
{[26] .}\end{array}$ \\
\hline Bi-Gaussian & $\begin{array}{c}\text { COST } 207 \text { channel } \\
\text { models } \\
\text { [27-29]. }\end{array}$ & $\begin{array}{c}S(f)=\frac{1}{\sum_{i=1}^{2} A_{i}} \\
{\left[\sum_{i=1}^{2} \frac{A_{i}}{\sqrt{2 \pi \sigma_{i}^{2}}} \exp \left(-\frac{\left(f-f_{i}\right)^{2}}{2 \sigma_{i}^{2}}\right)\right]} \\
* \text { where } f_{i}, A_{i}, \text { and } \sigma_{i}, \\
\text { are center frequencies, } \\
\text { power gains, and } \\
\text { standard deviations, } \\
\text { respectively. }\end{array}$ & $\begin{array}{l}\text { Modeling of multipath } \\
\text { echoes of the terrestrial } \\
\text { propagations in urban } \\
\text { areas. }\end{array}$ \\
\hline Bell & $\begin{array}{c}\text { IEEE 802.11 TGN } \\
{[30] .}\end{array}$ & $\begin{array}{l}\quad S(f)=\frac{1}{1+A\left(\frac{f}{f_{d}}\right)^{2}} \\
\text { * where } A \text { is a constant, } \\
\text { used in order to define } \\
0.1 S(f) \text { at a given } \\
\text { frequency } f_{d}, \text { being the } \\
\text { Doppler spread }\left(|f| \leq f_{d}\right) \text {. }\end{array}$ & $\begin{array}{l}\text { Proposed for the indoor } \\
\text { MIMO channels. }\end{array}$ \\
\hline
\end{tabular}




\section{SCQIC ENCODER STRUCTURE}

The SCQIC encoder of our interest composed of the two recursive systematic convolutional (RSC) component encoders linked by quadratic interleaver. We give specifications of RSC component codes which are utilized in this study on SCQICs. Consider a $(L-1)^{2}$-state rate $R_{0}=1 / 2$ RSC outer code where $L$ denotes the code's constraint length. Denote an input at time $k$ by $d_{k}$, the first output pair $X_{k}$ is equal to $d_{k}$. Using feedback (FB) and feedforward (FF) polynomials $g^{(0)}(D)$ and $g^{(1)}(D)$, the remainder $r(D)$ can be found. The feedback variable is [31]:

$r_{k}=d_{k}+\sum_{j=1}^{(L-1)^{2}} r_{k-j} g_{j}^{(0)}$

and parity data $Y_{k}$ is:

$Y_{k}=\sum_{j=0}^{(L-1)^{2}} r_{k-j} g_{j}^{(1)}$

A 8 -state rate $R_{0}=1 / 2$ convolutional code as outer code with feedforward (FF) and feedback (FB) polynomial trellis structures of $\{1111,1101\}_{2}$ and $\{1111\}_{2}$ is used; For inner convolutional encoding, we utilized a 64-state rate $R_{i}=2 / 3$ convolutional code with $F F:\{1111,1101,000 ; 000,1111,1101\}_{2}$ and $F B:\{1111,1111\}_{2}$; hence, the rate $1 / 3$ coding scheme is employed which is vastly considered as a promising code rate for enabling effective operating point in balancing common demands for both power and bandwidth efficiencies (hence, this turbo-code's rate is referred to as global coding rate [32]).

In principle, the interleaver is used to break up the error bursts and spread them across several codewords. While the design of interleavers for parallel turbo codes has enjoyed significant attention, interleavers designed for the serial turbo codes remain scarce [5]. The most used type of interleaver in communication systems is the block interleaver, which can be described by a matrix wherein the block of $\left(N_{I N T} / 2\right)-$ bit data is randomized at bit-level by filling a matrix row by row and shifting out column by column. The block permutation function is given by $\pi(i+j \times R+1)=i \times C+j+1, \forall i \in I=$ $\{0,1, \ldots, R-1\}$ and $\forall j \in J=\{0,1, \ldots, C-1\}$, which implies that any two bits initially at a distance less than $\min (I, J)$ will be situated at a distance superior to $\min (I, J)$ after applying the permutation function [33]. Herein $R$ and $C$ are positive integers where $R \cdot C=N_{I N T}$. Although they have shown to result in the acceptable BER/FER system performance, the randomization mapping based on the Takeshita et al. algorithm is a potential performance enhancement strategy which suggests to permute a sequence of bits based on property of quadratic congruences over the ring of integers modulo powers of two [6]. Thus, the terms quadratic and Takeshita et al. interleaver are used interchangeably in this article. Let $\bar{v}=\left\langle v_{1}, v_{2}, \cdots, v_{N_{I N T}-1}\right\rangle$ be a sequence in $\{0,1\}^{N_{I N T}}$. An interleaver $\pi_{N_{I N T}}$ maps $\bar{v}$ to a sequence $\bar{w}=\left\langle w_{1}, w_{2}, \cdots, w_{N_{I N T}-1}\right\rangle$ such that $\bar{w}$ is a permutation of the elements of $\bar{v}$. i.e. the functionality of the permutor is described by invertible permutation function $\pi$ on the numbers $\left\{0,1, \ldots N_{I N T}\right\}$ so that $\pi:\left\{0,1, \ldots N_{I N T}\right\} \rightarrow\left\{0,1, \ldots N_{I N T}\right\}$. Let $\mathrm{C}=\{0$, $\left.\ldots, N_{I N T}-1\right\} ; \pi_{N_{I N T}}$ can be defined by one-to-one and onto index mapping function $d_{\pi_{N_{I N T}}}: \mathrm{C} \rightarrow \mathrm{C}, d_{\pi_{N_{I N T}}}: i \rightarrow j, i, j, \in \mathrm{C}$, and can be expressed as a set called the permutation vector $\bar{\pi}_{N}=$ $\left[d_{\pi_{N_{I N T}}}(0), d_{\pi_{N_{I N T}}}(1), \ldots, d_{\pi_{N_{I N T}}}\left(N_{I N T}-1\right)\right][6]$.
Theorem I. The function $(m) \equiv \frac{k m(m+1)}{2}\left(\bmod N_{I N T}\right)$, $0 \leq m<N_{I N T}, k$ an odd constant, is bijective for any $N_{I N T}$ equal to a power of 2 ; thus, we have a permutation $\beth_{N_{I N T} \text { :FN } N_{I N T}}$ with a single $N_{I N T}$-cycle if we define $d_{\beth_{N_{I N T}: F N_{I N T}}}$ as its index mapping function [6-7,34]:

$d_{\beth_{N: F N}}: F(m) \rightarrow F(m+1)(\bmod N), 0 \leq m<N_{I N T}$

Theorem II. In the permutation vector of a $\beth_{N_{I N T}: F N_{I N T}}$ interleaver, a shift of $\gamma=N_{I N T} / 2$, produces an interleaver whose permutation vector is composed of 1-cycles and 2-cycles [6].

Hence, for 16-bit permutation length, take $k=9$ where $k \in\{1,3,7,9,11,14,15\}$, the fundamental interleaver arrays are given as in Table II; for $\bar{v}=\langle 1110001010011000\rangle_{\left(N_{I N T}\right)}$, which

TABLE II. QUADRATIC PERMUTATIONS FOR $N=16$ FOR CYCLIC SHIFTS $\{1: 1: 15\}$.

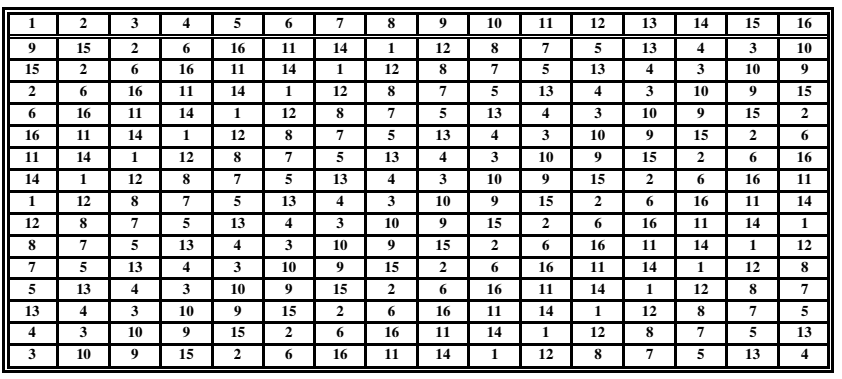

could be regarded as an arbitrary bit sequence of outer encoder output given that input bit sequence is $\langle 11011010\rangle_{\left(N_{I N T} / 2\right)}$, the corresponding randomized sequences are given as follows; wherein the upper and lower indices indicate the multiplicative factor and cyclic shift, respectively:

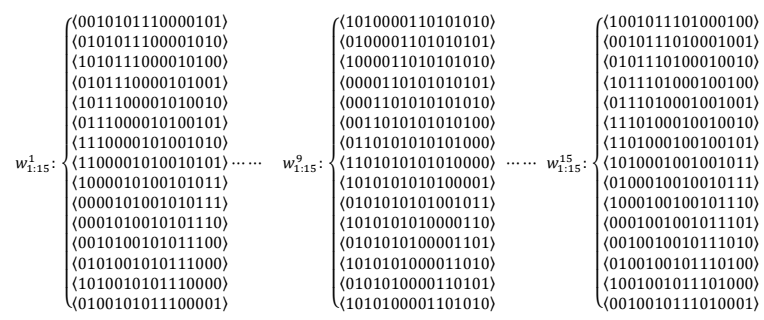

A given permuted sequence of length $N_{I N T}$ is inserted into inner encoder, resulting in $\left(3 \cdot N_{I N T}\right) / 2-$ bit coded sequence.

\section{NEAR-ML DECODING OF SCQICS}

The detected 8-DPSK symbol alphabets are fed into SCQIC decoder for recovering the transmitted information bits; i.e. the received constellations presented as I/Q components are demodulated and differentially decoded. The block diagram for the SCQIC decoder is depicted in Fig. 2. The core of decoding process for the SCQICs is the Maximum a posteriori (MAP) probability decoding based on Bahl et al. algorithm [15]. The decoding could be performed by iterating the Bahl et al. algorithm on convolutional component codes corresponding to the codecs of the encoders; i.e. for the system inner decoder, a 
64-state soft-input and a soft-output turbo decoder with: $F F:\{1111,1101,000 ; 000,1111,1101\}_{2}$ and $F B:\{1111,1111\}_{2}$ is, and for outer decoder, a 8-state soft-input/-output decoder with $F F:\{1111,1101\}_{2}$ and $F B:\{1111\}_{2}$. While the message passed from the outer decoder as one of the inputs of inner decoder is randomized using the function described in previous section, the input of outer decoder from the output of inner decoder should utilize the inverse function of the permutor; i.e. the unscramble or deinterleaver. An example of the performing unscrambling function for quadratic permutations is given as follows; wherein the $w_{\aleph}$ is an arbitrary binary sequence of length $\aleph$ :

$$
\begin{aligned}
& \mathrm{W}_{8}=(10100111) \stackrel{\pi^{-1}}{\rightarrow}(01110110) \\
& W_{16}=(1010100111011010) \stackrel{\pi^{-1}}{\rightarrow}(1001111110001001) \\
& W_{32}=(10100111010100111011010100101001) \stackrel{\pi^{-1}}{\rightarrow}
\end{aligned}
$$

(11010110111010011101110001000100)

At every iteration, a single decoding is performed using the channel observations as well as the reliability information delivered by other component decoder that was acted before, known as extrinsic information, given by (4) as:

$L_{\left(d_{k} \mid y\right)}=\ln \left(\frac{P\left(d_{k}=+1 \mid \grave{y}\right)}{P\left(d_{k}=-1 \mid y\right)}\right)$

where $y$ presents the received symbol sequence. The polarity of the $L_{\left(d_{k} \mid y\right)}$ decides the transmitted bit $\left(d_{k}\right)$, and its amplitude gives a correct decoding level [35]. If the present state $s$ and the previous state $s$ are known in the decoding trellis diagram, the $d_{k}$ which has brought the transmission between these two states will be known; hence, (4) could be rewritten as (5):

$$
L_{\left(d_{k} \mid y\right)}=\ln \left(\frac{\sum_{\substack{(s, s) \\ d_{k}=+1}} P(\dot{s}, s, y)}{\sum_{\substack{(s, s) \\ d_{k}=-1}} P(s, s, y)}\right)
$$

Afterwards, the key operations in the SCQIC decoder utilizing the MAP algorithm (summarized in the flowchart of Fig. 3) are performed sequentially. The iterative process continues until a stopping criterion is fulfilled; several promising stopping criteria have been proposed based on the sign difference ration or cross entropy between the distributions of estimated outputs of the decoders at each iteration [36-37], or in other schemes [38] based on the signature code of estimated outputs.

\section{NUMERICAL RESUlTS AND DISCUSSIONS}

In order to provide a concrete demonstration on the outstanding coding gains of the SCQICs in different wireless multiple-antenna links, i.e. SISO/MISO/MIMO systems with the statistical channel characteristics described in section II. B; first in section A of this part, we begin with exemplifying the utilization of the SCQIC in wireless systems operating in the Bell and Gaussian Doppler environments, by reviewing our previous related outcomes in $[8,10,14]$. The major performance parameters we have employed in the numerical error-rate computations of $[8,10,14]$ are shown in Table III. Afterwards, in section $\mathrm{B}$ and $\mathrm{C}$, we will start by presenting comprehensives

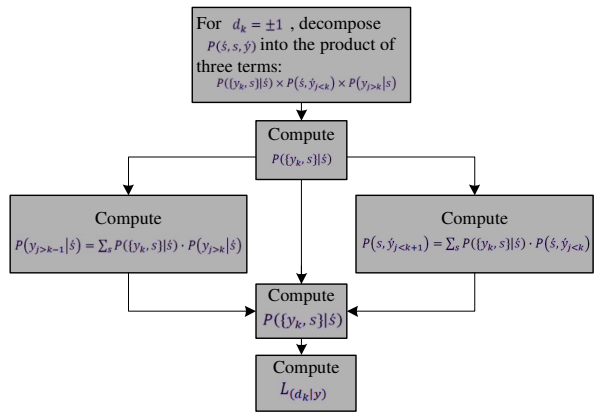

Figure 3. Key operations in iterative turbo decoder.

simulation results (i.e. the major scientific contributions of this article: items (2) and (3) of section I. B). A complete set of simulation results are presented in which the additional coding gains for provisioning $\mathrm{BER} \approx 10^{-5}$ due to utilizing quadratic interleavers are compared with the same FEC entity using the block permutations of $R \approx C \approx \sqrt{N_{I N T}}$ dimensions. Furthermore, in order to enable higher level of selection flexibility in errorrate versus computational complexity trade-off for the SCQICs (one of the major design issues in theory and practice for turbo and turbo-like codes), we will examine the required $E_{b} / N_{0}$ to attain $\mathrm{BER} \approx 10^{-5}$ for the discussed system models, employing original and approximated near maximum-likelihood decoders.

\section{A. A Review on the Major Outcomes of [8,10,14]}

Example 1: In [8], we considered the initial performance evaluation framework for LoS SISO/MISO generic femtocell links employing systematic SCQICs; herein, we examined their BER performance from low-to-medium SNRs for assessing the coding gain and comparing the results with the same system specifications as in SCQICs, but employing the conventional block scrambler which is the most-used permutation scheme in communication systems. In Fig. 4, we present the summary of the major comparisons. The study suggests that when one considers the coding gains on the average of $\approx 1.5-2 \mathrm{~dB}$ with moderate operating SNR (i.e. mostly occurs for LoS scenarios where $K_{r}>0$ ), SCQICs offer provisioning energy efficiency. Compared with superiority of SCQICs in enabling considerable low $E_{b} / N_{0}$ with reduced complexity [7], the block-interleaved schemes results in inefficient inferior performance of up to $\approx 2.5 \mathrm{~dB}$ as the depth and span follows either $R \ll C$ or $R \gg C$; this significant deterioration could be compensated when $R, C \rightarrow \sqrt{N_{I N T}}$; e.g. for representative permutor length $N_{I N T}=$ 256 , there exist energy gaps of up to $3 d B$ when dimensions are configured as $R, C=16$ rather than $R, C \in\{2,128\}$.

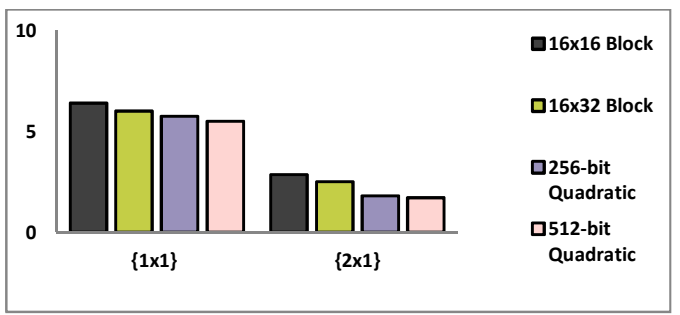

Figure 4. Comparison of the required $E_{b} / N_{0}$ for attaining $\mathrm{BER} \approx 10^{-4}$ for the SCICs employing block and quadratic permutors. 
Example 2: In [10], we characterized the performance of SCQICs in conjunction with Tarokh et al.'s orthogonal spacetime block codes (OSTBCs) [12], which have evolved at an exceptional rate during the past decade and reached a state of maturity in theory after their breakthrough discovery so that they quickly found their way into the practical applications and standards for enabling outstanding reliable communications; such as, e.g. GSM/UMTS/LTE wireless LANs (WLANs) [39], Wireless Mesh Networks (WMNs) [40], mobile WiMAX 802.16 [41], and RFID systems [42]. As far as the $M=16-$ QAM modulation scheme provides a good trade-off in terms of the power and bandwidth efficiencies and therefore widely adopted in standardized wireless systems (e.g., DVB, HSDPA protocol, and IEEE 80211a PHY layer for modulating OFDM subcarriers), the introduced integrated OSTBC-SCQICs results in the significant coding gains while also improving spectral efficiencies. By investigating the error-rate of this system under the assumption that mobile terminals move the characteristics of deep urban environment (i.e. $K_{r} \rightarrow 0$ ), it is shown they result in lowering the operating SNR for more than over $3 d B$ for yielding $\mathrm{BER} \approx 10^{-4}$ and $10^{-5}$ when Alamouti's twin-antenna space-time codes and Tarokh et al.'s triplet-antenna OSTBCs are used, respectively.

Example 3: In the case of generic stochastic aeronautical channel, we have focused on employing SCQICs by initiating a work for developing them for the MISO wireless links with $K_{r}=\{1,4\}$ and Gaussian Doppler spectrum. Considering the scenarios wherein an arbitrary aircraft with the take-off and cruising velocities around $180 \mathrm{mph}$ and $565 \mathrm{mph}$, respectively; and taking into account the point that the spatial angle between the direction of motion of the aircraft and the direction of arrival of the wave has key role in determining the frequency dispersion, we define two arbitrary points wherein in one the speed of the aircraft is on average of take-off and also cruising velocities with the moderate angle of arrival (case-1); while in the other critical angular position, the arrival angle approaches 90 degrees and the speed of the aircraft is at the cruising stage (case-2). For the carrier frequency of $25 \mathrm{MHz}$, the Doppler frequencies considered to be $f_{d}<100 \mathrm{~Hz}$ and $\ll 100 \mathrm{~Hz}$, for the first and second flight scenarios, respectively. The performance evaluation demonstrations recommend that space-time SCQICs are adaptable to the peculiarities of the airport LoS scenarios in the Aeronautical Mobile Airport Communications Systems (AeroMACS) [43]; since in these scenarios, the $K_{r}$ tends to be greater and not equal to zero. As the reliable and accurate navigation of vehicles in AeroMACS is of crucial importance, space-time SCQICs in conjunction with deployment of two antennas at the transmitting part of AeroMACS result in good compromise (with respect to the important performance factors summarized in Fig. 1) between coding gain, error-rate, overall complexity, and channel characteristics; i.e. using $N_{I N T}<1000$ bits, for the case of worst channel condition (which is $K_{r} \approx 1$ for the case- 2 frequency dispersion conditions), and while also using the aforementioned advantages of the space-time SCQIC schemes, and enjoying the flexible selection between the coherent/incoherent detection; it has been shown that the proposed bit-level space-time FEC enables the satisfactory wireless communications reliability of $\log (B E P) \approx-4$ for the operating SNR of no more than $\approx 7.5 \mathrm{~dB}$.
TABLE III. MAIN PERFormanCE PARAMETERS USED IN THE NUMERICAL ERROR-RATE COMPUTATIONS OF $[8,10,14]$.

\begin{tabular}{|c|c|c|c|c|}
\hline & [8] & [10] & [14] \\
\hline \multirow{6}{*}{ 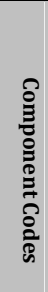 } & RSC & $\checkmark$ & $\checkmark$ & $\checkmark$ \\
\hline & $\mathbf{L}$ & 4 & 3 & 3 \\
\hline & $\mathbf{R}_{\text {out }}$ & $1 / 2$ & $1 / 2$ & $1 / 2$ \\
\hline & $\mathbf{R}_{\text {in }}$ & $2 / 3$ & $2 / 3$ & $2 / 4$ \\
\hline & $\mathbf{G}_{\mathbf{0}}$ & 17 & 7 & 7 \\
\hline & $\mathrm{G}_{1}$ & 13 & 5 & 5 \\
\hline \multicolumn{2}{|c|}{$\begin{array}{l}\text { Permutor } \\
\text { Length }\end{array}$} & $\begin{array}{l}N_{I N T} \\
=\{128,256,512\}\end{array}$ & $N_{I N T}=1024$ & $N_{I N T}=1024$ \\
\hline \multicolumn{2}{|c|}{$\begin{array}{l}\text { Decoding } \\
\text { Algorithm }\end{array}$} & Log - MAP & $\begin{array}{l}\text { MAP and Max - Log } \\
\text { - MAP }\end{array}$ & Max - Log - MAP \\
\hline \multicolumn{2}{|c|}{ Modulation } & BPSK (coherent) & 16 - QAM (coherent) & $\begin{array}{l}\text { Differential BPSK, Gray } \\
\text { \& Binary coded coherent QPSK }\end{array}$ \\
\hline \multicolumn{2}{|c|}{ Channel } & $\begin{array}{l}\text { Rician SISO and } 2 \times 1 \\
\text { MISO }\left(K_{r}=12\right) \text { with } \\
\text { AWGN at the Rx, and } \\
\text { Bell Doppler spectrum }\end{array}$ & $\begin{array}{l}\text { Rayleigh } 2 \times 1 \text { MISO and } \\
3 \times 2 \text { MIMO and Bell } \\
\text { Doppler spectrum }\end{array}$ & $\begin{array}{l}\text { Rician MISO }\left(K_{r}=1 \text { and } 4\right) \\
\text { with AWGN at the Rx, and } \\
\text { Gaussian Doppler spectrum }\end{array}$ \\
\hline
\end{tabular}

\section{B. Performance Predictions in Different Wireless Doppler Environments}

For 512-bit message frames with Bernouli distribution, i.e. with the probability mass function (PMF) $f(k ; p)=p$ if $k=1$, $(1-p)$ if $k=0$, and 0 otherwise, in Fig. 5-8, the required $E_{b} / N_{0}$ to attain the BER $\approx 10^{-5}$ for SCQIC FECs of section III and IV are presented. As far as Doppler spectrum and $E_{b} / N_{0}$ are important parameters for assessing the quality and rate of change of wireless channels [44], energy-gap computation and comparison for the discussed channel conditions are provided. Under the assumption that the calculations of $E_{b} / N_{0}$ take $90 \%$ of confidence level when computing the error-rates, the mean average coding gain gap (MACGG) between SCQIC-coded systems and block-interleaved systems with $R \approx C \approx \sqrt{N_{I N T}}$ for four aforementioned Doppler PSDs are (in order of Rounded, Bell, Gaussian, and Bi-Gaussian) 1.05, 1.2, 0.93, and $1.65 \mathrm{~dB}$ for SISO, $1.5,1.25,0.625$, and $0.7 d B$ for MISO (2Tx-1Rx), $1.325,1.325,0.65$, and $0.525 d B$ for MIMO (2Tx-2Rx), and $1.17,1.17,0.4$, and $0.4 d B$ for MIMO (3Tx-3Rx). Based on the firm performance predictions discussed in Example 1, we can predict that additional gain of $\approx 2.5 \mathrm{~dB}$ is already attained since block permutor is configured as $R, C \rightarrow \sqrt{N_{I N T}}$.

Using the same parameters, for iteratively exchanging the intermediate results (extrinsic information given in (4)) between soft-input/-output component decoders, optimal and suboptimal MAP algorithms are employed at the core of the SCQIC decoder architecture (Fig. 9). For enabling suboptimal MAP decoding, the three decomposed terms of (5) could be calculated in logarithmic domain using the Jacobian logarithm [17]; hence, in order to preserve the MAP algorithm and yield equivalent performance of the original Bahl et al. method, all maximizations can be augmented over the two values with the correction function, which is the maximum of the function's two arguments plus a nonlinear correction function that is only a function of the absolute difference between the two arguments. As a result, the extrinsic information in equation (5) can be derived in terms of new metrics that save considerable 
computational complexities as discussed in [45]. Following the fact that using the Max-Log-MAP algorithm's approximations result in the significant computational complexity savings for the entire FEC encoding/decoding process, as discussed and presented comprehensively in [46-47], the sacrificed coding gain $\approx 0.5 d B$ reveals that for minimizing bit-error-probability of the received SCQIC-encoded message bits, Max-Log-MAP algorithm is equivalent to the true Bahl et al. MAP, but without its major disadvantage of the computational complexities; i.e. based on the analysis of [45] which expressed the complexity comparison of approximated MAP algorithms in terms of the number of equivalent additions, we computed and found the overall complexity of Max-Log-MAP to be at least 1.64 times lower than the original MAP (which will be higher for larger constraint lengths; e.g. 1.66, 1.69, and for the $L=4$ and 5, respectively).

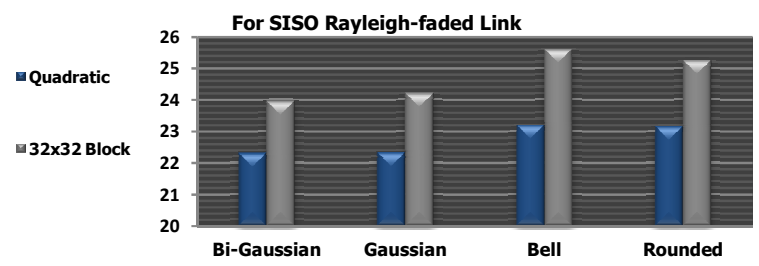

Figure 5. Comparison of the required $E_{b} / N_{0}$ for attaining BER $\approx 10^{-5}$ for SCQICs vs. SCICs employing block permutors.

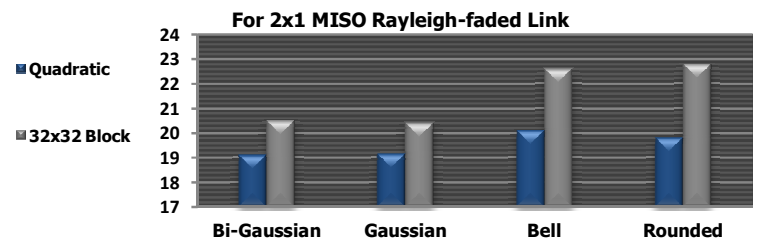

Figure 6. Comparison of the required $E_{b} / N_{0}$ for attaining BER $\approx 10^{-5}$ for Alamouti's space-time coded SCQICs vs. SCICs employing block permutors.

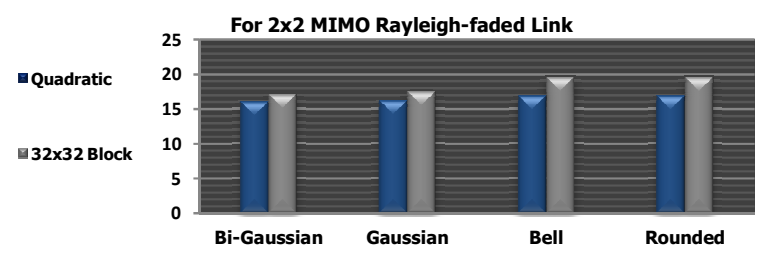

Figure 7. Comparison of the required $E_{b} / N_{0}$ for attaining BER $\approx 10^{-5}$ for Alamouti's space-time coded SCQICs vs. SCICs employing block permutors.

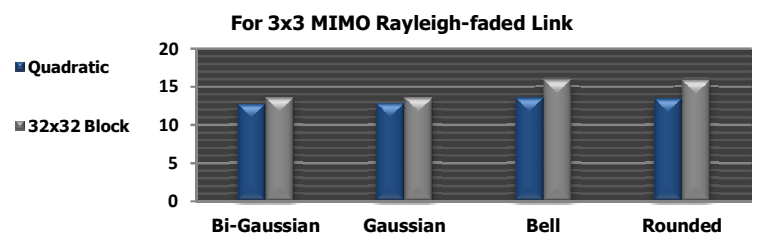

Figure 8. Comparison of the required $E_{b} / N_{0}$ for attaining BER $\approx 10^{-5}$ for Tarokh et al.'s OSTBC-coded SCQICs vs. SCICs employing block permutors.

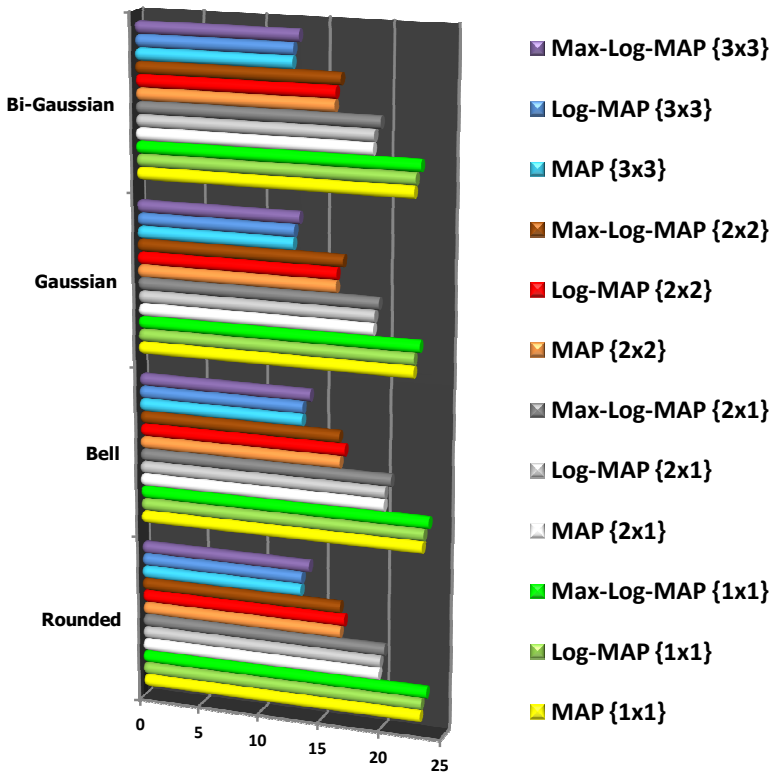

Figure 9. Comparison of the required $E_{b} / N_{0}$ for attaining $\mathrm{BER} \approx 10^{-4}$ for the original and approximated MAP-based SCQIC decoders, employed in conjunction with space-time ML detector and estimator of [9] for $\{2 \times 1\}$ and $\{2 \times 2\}$ schemes, and OSTBC detection technique of [11] for $\{3 \times 3\}$ transmissions.

\section{CONCLUDING REMARKS}

In this article, we have invoked Takeshita et al. algorithm for construction of permutation functions with nearly the same statistical distribution as a randomly chosen interleavers [6], while also enable the straightforward implementation [7]; their performances in the various single-/multiple-antenna wireless Doppler environments have been presented and shown to withstand the severe wireless channel conditions (as discussed in detail), and result in provisioning MACGG compared with their block interleaved counterparts even in the case that the block configures with its optimum dimensions with respect to the error-rate performance factor, i.e. $R, C \rightarrow \sqrt{N_{I N T}}$. We also demonstrated a significant benefit of employing approximated variants of Bahl et al.'s original soft-decision MAP decoding, and presented that they yield equivalent error-rate to the original scheme, while also reduce the incurred iterative turbo decoder computational complexities.

\section{REFERENCES}

[1] C. Berrou, A. Glavieux, and P. Thitimajshima, "Near Shannon limit error-correcting coding and decoding: turbo codes," IEEE ICC, Jun. 1993, pp. 1064-1070.

[2] C. Berrou and A. Glavieux, "Near optimum error correcting coding and decoding: Turbo codes," IEEE Trans. Commun., vol. 44, no. 10, Oct. 1996, pp. 1261-1271.

[3] S. Benedetto, D. Divsalar, G. Montorsi, and F. Pollara, "Serial concatenation of interleaved codes: Performance analysis, design, and iterative decoding," IEEE Trans. Inf. Theory, vol. 44, pp. 909-926, May 1998.

[4] S. Benedetto and G. Montorsi, "Serial concatenation of block and convolutional codes,” Electron. Lett., vol. 32, pp. 887-888, 1996. 
[5] R. G. Maunder and L. Hanzo, "Evolutionary algorithm aided interleaver design for serially concatenated codes," IEEE Trans. Commun., vol. 59, no. 6, Jun. 2011.

[6] O. Y. Takeshita and D. J. Costello, "New classes of algebraic interleavers for turbo-codes," IEEE ISIT, Aug. 1998.

[7] O. Y. Takeshita and D. J. Costello, "New deterministic interleaver designs for turbo codes, "IEEE Trans. Inf. Theory, vol. 46, no. 6, pp. 1988-2006, Sep. 2000.

[8] F. Mehran and A. Rahimian "Physical layer performance enhancement for femtocell SISO/MISO soft real-time wireless communication systems employing serial concatenation of quadratic interleaved codes," $20^{\text {th }}$ Iranian Conf. Elect. Eng., May 2012, pp. 1188-1193.

[9] S. M. Alamouti, "A simple transmit diversity technique for wireless communications," IEEE J. Sel. Areas Commun., vol. 16, no. 8, pp. 1451-1458, Oct. 1998.

[10] A. Rahimian and F. Mehran, "BEP enhancement for semi-femtocell MIMO systems employing SC-QICs and OSTBCs," Int. J. Electronics Commun. and Comput. Technol. (IJECCT), vol. 3, no. 1, pp. 329-332, Jan. 2013.

[11] V. Tarokh, H. Jafarkhani, and A. Calderbank, "Space-time block coding for wireless communications: performance results," IEEE J. Sel. Areas Commun., vol. 17, pp. 451-460, Mar. 1999.

[12] V. Tarokh, H. Jafarkhani, and A. Calderbank, "Space-time block codes from orthogonal designs," IEEE Trans. Inf. Theory, vol. 45, pp. 14561467, Jul. 1999

[13] F. Mehran, "Performance evaluation of serial concatenated convolutional codes over aeronautical channels," $5^{\text {th }}$ Int. Symp. Telecommun. (IST), Dec. 2010, pp. 361-366.

[14] A. Rahimian and F. Mehran, "Short-length FSU-SCQICs over coherent and incoherent stochastic aeronautical MISO channels," $19^{\text {th }}$ AsiaPacific Conf. Commun. (APCC), Aug. 2013.

[15] L. R. Bahl, J. Cocke, F. Jelinek, and J. Raviv, "Optimal decoding of linear codes for minimizing symbol error rate," IEEE Trans. Inf. Theory, vol. 22, pp. 284-287, Mar. 1974.

[16] P. Robertson, E. Villebrun, and P. Hoeher, "A comparison of optimal and sub-optimal MAP decoding algorithms operating in the log domain," IEEE ICC, Jun. 1995, vol.2, pp. 1009-1013.

[17] J. Erfanian, S. Pasupathy, and G. Gulak, "Reduced complexity symbol detectors with parallel structures for ISI channels," IEEE Trans. Commun., vol. 42, pp. 1661-1671, Feb./Mar./Apr. 1994.

[18] T. H. Liew and L. Hanzo, "Space-time codes and concatenated channel codes for wireless communications," Proc. of the IEEE, vol. 90, no. 2, pp. 187-219, Feb. 2002

[19] L. Hanzo and B. Y. T. H. Liew. Turbo Coding, Turbo Equalisation and Space-Time Coding, New York: Wiley-IEEE Press, 2002.

[20] F. Gini and G. B. Gianna, "Generalized differential encoding: a nonlinear signal processing framework," First IEEE Workshop on Signal Process. Advances in Wireless Commun., 1997, pp. 153-156.

[21] R. F. H. Fischer, et al., "Coded modulation for differential encoding and non-coherent reception on fading channels," Int. ITG Conf. on Systems, Commun. and Coding (SCC), Jan. 2000, pp. 139-146.

[22] A. F. Molisch, Wireless Communications, $2^{\text {nd }}$ Edition, John Wiley \& Sons Ltd., Wiley-IEEE Press, 2011.

[23] C. Q. Xu, C. L. Law, and S. Yoshida, "On the Doppler power spectrum at the mobile unit employing a directional antenna," IEEE Commun. Lett., vol. 5, no. 1, pp. 13-15, Jan. 2001.

[24] IEEE 802.16 Broadband Wireless Access Working Group, "Channel models for fixed wireless applications," IEEE 802.16a-03/01, 2003

[25] ANSI J-STD-008, Personal Station-Base Station Compatibility Requirements for 1.8 to $2.0 \mathrm{GHz}$ Code Division Multiple Access (CDMA) Personal Communications Systems, Mar. 1995.

[26] P. Bello, "Aeronautical channel characterization," IEEE Trans. Commun., vol. 21, no. 5, pp. 548-563, May 1973.

[27] M. Failli, "Digital land mobile radio communications," COST 207 Final Rep., Sep. 1989.
[28] Jean-Paul M. G. Linnartz, "Properties of the mobile radio propagation channel", Dep. Head CoSiNe, Nat. Lab., Philips Research. Available online: www.smsmatrix.com/front/mob_doc/tech-1/ppt/prop2.ppt

[29] M. Patzold, Mobile Fading Channels, UK: Wiley, 2002.

[30] V. Erceg, et al., "TGn Channel Models," IEEE P802.11 Wireless LANs Technical Report, May 2004.

[31] N. Odabasioglu, A. K. Tanc, and O. N. Ucan, "2D image transmission using Kalman-turbo systems," Istanbul Uni. J. Elect. and Electronics Eng., vol. 5, no. 2, pp. 1443-1448, Feb. 2005.

[32] C. Chi, "Quadratic permutation polynomial interleavers for LTE turbo coding," Int. J. Future Comput. Commun., vol. 2, no. 4, pp. 364-367, Aug. 2013.

[33] M. Kovaci, H. G. Balta, and M. M. Nafornita, "The performances of interleavers used in turbo codes," Int. Symp. on Signals, Circuits, and Systems (ISSCS), Jul. 2005, vol. 1, pp. 363-366.

[34] C. Heegard and S. B. Wicker, Turbo Coding, Kluwer Academic Publishers, 1999.

[35] W. Gu, L. Clavier, N. Rolland, and P. Rolland, "Turbo code decoding in MAI environment," $6^{\text {th }}$ Int. Symp. on Turbo Codes and Iterative Inf. Process. (ISTC), Sep. 2010, pp. 206-210.

[36] Y. Rose, et al. "Two simple stopping criteria for turbo decoding," IEEE Trans. Commun., vol. 47, no. 8, pp. 1117-1120, Aug. 1999.

[37] Y. Wu, B. Woemer, and J. Ebel, "A simple stopping criterion for turbo decoding," IEEE Commun. Lett., vol. 4, no. 8, pp. 258-260, Aug. 2000.

[38] T. M. N. Ngatched and F. Takawira, "Simple stopping criterion for turbo decoding," Electron. Lett, vol. 37, no. 22, pp. 1350-1351, Oct. 2001.

[39] X. Zhou, X. Quan, and R. Li, "A dual-broadband MIMO antenna system for GSM/UMTS/LTE and WLAN handset," IEEE Antennas Wireless Propag. Lett., vol. 11, pp. 551-554, May 2012.

[40] W. Jaafar, W. Ajib, and S. Tabbane, "The capacity of MIMO-based wireless mesh networks," Proc. IEEE Int. Conf. on Netw. (ICON), Nov. 2007, pp. 259-264.

[41] Q. Li, X. Lin, J. Zhang, and W. Roh, "Advancement of MIMO technology in WiMAX: from IEEE $802.16 \mathrm{~d} / \mathrm{e} / \mathrm{j}$ to $802.16 \mathrm{~m}$," IEEE Commun. Mag., vol. 47, no. 6, pp. 100-107, Jun. 2009.

[42] R. Langwieser, C. Angerer, and A. L. Scholts, "A UHF frontend for MIMO applications in RFID," Proc. IEEE Radio and Wireless Symp. (RWS), pp. 124-127, Jan. 2010.

[43] J. M. Budinger and E. Hall, Fututre Aeronautical Communications, Chapter 12: Aeronautical Mobile Airport Communications System (AeroMACS), S. Plass, Ed., InTech, 2011.

[44] A. Dogandzic, et al., "Estimating Jakes' doppler power spectrum parameters using the Whittle approximation," IEEE Trans. Signal Process., vol. 53, no. 3, pp. 987-1005, Mar. 2005.

[45] I. A. Chatzigeorgiou, et al., "A comparison of convolutional and turbo coding schemes for broadband FWA systems", IEEE Trans. Broadcast., vol. 53, no. 2, pp. 494-503, Jun. 2007.

[46] B. Vucetic and J. Yuan, Turbo Codes: Principles and Applications, Kluwer, Academic Publishers, 2000.

[47] H. R. Sajadpour, "Maximum a posteriori decoding algorithms for turbo codes," Proc. SPIE, vol. 4045, pp. 73-83, Jul. 2000. 